\title{
Water Solubilization and Thermal Stimuli-Triggered Release of Porphyrin Derivatives Using Thermoresponsive Polysaccharide Hydroxypropyl Cellulose for Mitochondria-Targeted Photodynamic Therapy
}

\author{
Riku Kawasaki, ${ }^{*}+\underset{*}{+}$ Keita Yamana, ${ }^{\ddagger}$ Risako Shimada, Kouta Sugikawa, and Atsushi Ikeda*
}

Cite This: ACS Omega 2021, 6, 3209-3217

Read Online

\section{ACCESS |}

Wl Metrics \& More

Article Recommendations

Supporting Information

ABSTRACT: With minimal invasiveness and spatiotemporal therapeutic effects, photodynamic therapy is one of the most elegant strategies for achieving effective tumor therapy. Herein, a facile preparation and thermal process-triggered release of water-soluble photosensitizer 5,10,15,20-tetrakis(4-hydroxyphenyl)porphyrin (THPP) has been developed using a thermoresponsive polysaccharide, hydroxypropyl cellulose. Current systems using hydroxypropyl cellulose enable manipulation of the loading capacity of THPP into a polymer matrix and the size of the complex by varying the temperature of the solution in preparation. Furthermore, current systems have enabled the release of THPP using a heating process, mimicking the surrounding of mitochondria, and have resulted in THPP potency as a mitochondria-targeted photodynamic therapy.

\section{INTRODUCTION}

With minimal invasiveness and spatiotemporal therapeutic effects, photodynamic therapy (PDT) is a promising approach for the treatment of cancer and rheumatoid diseases. ${ }^{1,2}$ PDT is attained using reactive oxygen species (ROS) that are generated by photosensitizers, ${ }^{3}$ such as fullerenes, ${ }^{4}$ porphyrins, ${ }^{5}$ or phthalocyanine ${ }^{6}$ via irradiation with light of an optimal wavelength. In particular, porphyrin derivatives have been widely studied owing to their excellent absorbance of visible light $(600-800 \mathrm{~nm}) .^{5}$ The use of this range of wavelength is advantageous for obtaining efficient therapeutic efficacy via PDT because the light can reach deeper tissues in the body. ${ }^{7}$ Despite their clinical potencies, the application of porphyrin derivatives as photosensitizers has been limited because of poor water solubility and instability in water. ${ }^{8}$ From this perspective, the development of water-solubilizing techniques and platforms has been desired to expand the bioavailability of porphyrin derivatives. ${ }^{9}$

The conjugation of hydrophilic compounds, such as polyethylene glycol and saccharides, has been employed to improve the water solubility of porphyrin derivatives. ${ }^{10}$ However, chemical modification often causes an undesirable decline in photodynamic activity. ${ }^{11}$ To address this issue, approaches based on supramolecular chemistry, especially host-guest interactions, are powerful means to manipulate the water solubility of porphyrin derivatives. ${ }^{12}$ In particular, natural product-based agents, including cyclodextrins, ${ }^{13}$ polysaccharides, ${ }^{14,15}$ and liposomes, ${ }^{16}$ are promising candidates as water solubilizers because of their excellent biocompatibility.
Mitochondria-targeted delivery is one of the most robust approaches to therapeutic efficacy using $\mathrm{PDT}^{17}$ because oxidative stress on mitochondria leads to apoptosis in the treated cells. $^{18}$

Recently, various types of mitochondria-targeting nanocarriers have been developed using triphenylphosphonium ${ }^{19}$ signal peptides ${ }^{20}$ and thermoresponsive nanomaterials. ${ }^{21}$ In particular, thermoresponsiveness is advantageous in cancer therapy compared to other systems because cancer cells are maintained at higher temperatures than normal cells, including macrophages and endothelial cells. ${ }^{22}$

Herein, we have developed a facile mitochondria-targeted delivery platform using a hydrophobic porphyrin derivative, 5,10,15,20-tetrakis(4-hydroxyphenyl)porphyrin (THPP), and hydroxypropyl cellulose (HPC) for PDT because of responsiveness to thermal stimuli (Figure 1). To the best of our knowledge, our system is the first example to prove the availability of HPC as mitochondria-targeted delivery platforms for PDT. In addition, water-solubilization techniques using HSVM enable the dissolution of hydrophobic compounds in water without any complicated chemical modifications and harmful organic solvents. ${ }^{13}$

Received: November 20, 2020

Accepted: December 30, 2020

Published: January 19, 2021 


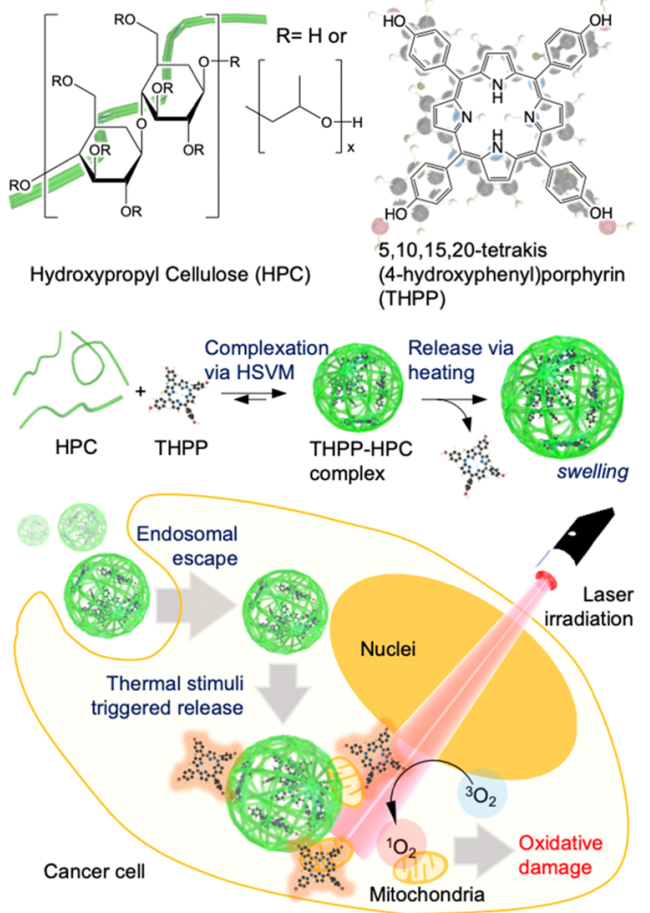

Figure 1. Schematic illustration of the water solubilization of ,10,15,20-tetrakis(4-hydroxyphenyl)porphyrin using hydroxypropyl cellulose via high-speed vibration milling (HSVM) and photodynamic activity against cancer cells.

With high biocompatibility, excellent solubility in both polar organic solvents and water, and responsive thermal stimuli, $\mathrm{HPC}$ is an attractive polysaccharide for constructing thermal stimuli-responsive biomaterials as drug-delivery systems ${ }^{23}$ and hydrogels. ${ }^{24}$ In particular, thermal stimuli-responsive materials are powerful means to deliver bioactive molecules to the mitochondria ${ }^{21}$ because the temperature of the mitochondria is estimated to be approximately $50{ }^{\circ} \mathrm{C} .{ }^{25}$ Additionally, HPC has been clinically used in ophthalmic inserts that are an effective and safe treatment for dry eye syndrome. ${ }^{26}$ Their amphiphilic properties are helpful for encapsulation of hydrophobic compounds mainly via hydrophobic interactions in water. These advantageous features of HPC encouraged us to develop delivery platforms with thermal stimuli-triggered release of encapsulated THPP by responding at a physiologically relevant temperature targeted to the mitochondrial microenvironment.

Here, we have developed mechanochemical HSVM techniques using oligo- and polysaccharides for water solubilization. ${ }^{27}$ HSVM techniques enable the solubilization of hydrophobic compounds, including fullerenes, ${ }^{28}$ porphyrins, ${ }^{29}$ and chlorines ${ }^{15}$ in water. In addition, the hydrophobic compounds complexed with oligosaccharides and polysaccharides exhibit excellent photochemical properties in water for bioimaging and photodynamic activities against cancer cells.

In this study, we have prepared a complex of THPP with HPC using HSVM techniques to develop mitochondriatargeted delivery platforms with thermal stimuli-triggered release systems for mitochondria-targeted PDT. With increasing temperature of the solvents during extraction, smaller nanoaggregations with greater THPP-loading capacity were formulated using HSVM. Furthermore, smaller complexes enhanced photoinduced cytotoxicity in cancer cells using irradiation with an optimal wavelength of light for PDT and with excellent deliverability of THPP to mitochondria.

\section{RESULTS AND DISCUSSION}

Basic Characterization of HPC. Initially, we estimated the degree of substitution of hydroxypropyl groups with glucose units in cellulose using ${ }^{1} \mathrm{H}-\mathrm{NMR}$ (Figure $\mathrm{S} 1$ ) as a basic characterization of HPC. The integral ratio of methyl groups in hydroxypropyl groups $(\delta, 0.98-1.03 \mathrm{ppm})$ to anomeric protons in cellulose backbones $(\delta, 4.0-4.8 \mathrm{ppm})$ was determined to be 21.67, indicating that the degree of substitution of hydroxypropyl groups was 722 per 100 glucose units. In addition, the lower critical solution temperature (LCST) value of HPC in water was determined to be $55^{\circ} \mathrm{C}$, as measured by the transmittance of the solution (Figure S2).

Preparation of the THPP-HPC Complex. The complexes of THPP with HPC were prepared using the HSVM technique, as previously reported. ${ }^{\mathrm{f}} \mathrm{We}$ used pullulan as a thermal stimuli-nonresponsive polysaccharide. The loading capacity of THPP using HPC and pullulan at various solvent extraction temperatures $\left(4,10,25,40\right.$, and $\left.55{ }^{\circ} \mathrm{C}\right)$ was measured. These THPP-HPC complexes were described as THPP-HPC-4, THPP-HPC-10, THPP-HPC-25, THPP-HPC40 , and THPP-HPC-55, respectively. In the case of THPPHPC-55, the largest amounts of THPP were dissolved in water (Figure S3). The hydrophobicity of the hydroxypropyl groups on HPC was increased by applying solvent at high temperatures, resulting in larger amounts of THPP dissolved in water. These results suggest that the HPC system enables the manipulation of the loading capacity of THPP within the polymer matrix by simply varying the extraction solution temperature. We further examined the water solubilization of THPP using HPC via a conventional sonication method. In the sonication method, it was tough to acquire suitable absorption spectra of the resulting dispersion (Figure S3 black line). This result suggests that our HSVM techniques are a powerful means to dissolve hydrophobic compounds in water.

Dynamic light scattering (DLS) measurement revealed that the hydrodynamic diameters of THPP-HPC were gradually decreased from 300 to $230 \mathrm{~nm}$ with the increasing temperature of the extraction solution, indicating a more hydrophobic condition of the side chains of HPC and the formulation of smaller nanoaggregates in the aqueous media (Table 1 ). The $\zeta$ -

Table 1. Solution Properties of the THPP-HPC Complex with Alternating Temperature in Preparation

\begin{tabular}{|c|c|c|c|c|c|}
\hline & $T /{ }^{\circ} \mathrm{C}$ & $\begin{array}{c}\text { THPP/ } \\
\mu \mathrm{M}\end{array}$ & $D_{\text {hy }} / \mathrm{nm}$ & PDI & $\begin{array}{c}\zeta \text {-potential/ } \\
\mathrm{mV}\end{array}$ \\
\hline $\mathrm{HPC}$ & & & $31 \pm 1$ & 0.61 & $-3.0 \pm 0.8$ \\
\hline ТНРP-НРС-4 & 4 & 16.9 & $300 \pm 8$ & 0.36 & $-10.8 \pm 0.1$ \\
\hline ТНPP-HPC-10 & 10 & 4.2 & $275 \pm 9$ & 0.39 & $-8.5 \pm 1.6$ \\
\hline THPP-HPC-25 & 25 & 8.8 & $265 \pm 3$ & 0.39 & $-7.4 \pm 0.4$ \\
\hline ТНРP-НРС-40 & 40 & 9.1 & $244 \pm 7$ & 0.13 & $-2.6 \pm 0.2$ \\
\hline THPP-HPC-55 & 55 & 30 & $230 \pm 2$ & 0.17 & $-9.3 \pm 0.2$ \\
\hline
\end{tabular}

potential did not significantly change upon the complex formation with HPC. Furthermore, morphological observation by transmission electron microscopy (TEM) revealed spherical structures (Figure 2a,b), and the average size of the complex (THPP-HPC-4, $320 \mathrm{~nm}$; THPP-HPC-55, $205 \mathrm{~nm}$ ) corresponded to the results from DLS measurements (Figure S4). In the case of non-thermoresponsive pullulan, the water 
(a)
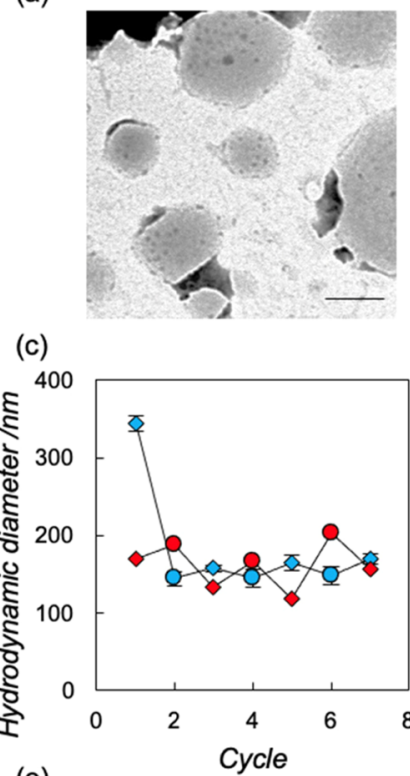

(e)
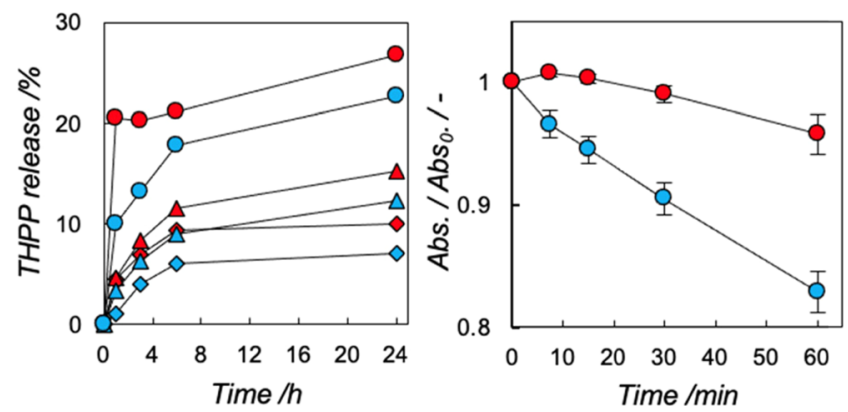

Figure 2. Characterization of the THPP-HPC complex. Representative morphology of the THPP-HPC complex in TEM ((a): THPPHPC-4; (b): THPP-HPC-55). Scale bars indicate $100 \mathrm{~nm}$. (c) Changes in the hydrodynamic diameter of THPP-HPC-4 (circle) and THPP-HPC-55 (diamond) during the heating/cooling thermal cycling from $37^{\circ} \mathrm{C}$ (blue) to $55^{\circ} \mathrm{C}$ (red). (d) Change in fluorescence during heating/cooling thermal cycling from $25{ }^{\circ} \mathrm{C}$ (blue) to $55{ }^{\circ} \mathrm{C}$ (red). (e) Thermal stimulus-triggered release of THPP complexes. THPP-HPC-4 (blue) or THPP-HPC-55 (red) were incubated in a medium containing $10 \% \mathrm{FBS}$ at $4{ }^{\circ} \mathrm{C}$ (diamond), $37{ }^{\circ} \mathrm{C}$ (triangle), or $50{ }^{\circ} \mathrm{C}$ (circle), and the fluorescence intensity of the released THPP was measured with a fluorometer. (f) Detection of the generated oxygen singlets using anthracene derivatives. The THPP-HPC-4 (blue) or THPP-HPC-55 (red) complex (THPP, $5 \mu \mathrm{M}$ ) was coincubated with 9,10-anthracenediyl- bis(methylene)dimalonic acid (ABDA). The samples were exposed to an optimal wavelength of light $\left(5 \mathrm{~W} \bullet \mathrm{cm}^{-2},>620 \mathrm{~nm}, 60 \mathrm{~min}\right)$. The data were presented as mean \pm $\mathrm{SD}(n=3)$.

solubility of THPP (Figure S5) and the hydrodynamic diameters of the complexes did not significantly change by varying the temperature of the extraction solvent (Table S1). The results from pullulan experiments support our hypothesis that the complex-formation behavior of HPC is highly dependent on the changes in the hydrophobicity of HPC.

Characterization of the THPP-HPC Complex. The thermoresponsiveness of the THPP-HPC complex was investigated, and the following experiments were conducted using THPP-HPC-4 and THPP-HPC-55. Initially, we addressed the size changes of the complexes during the thermal process by measuring DLS. THPP-HPC-4 and THPP-
HPC-55 were treated with repeated cycles of heating and cooling from 37 to $60{ }^{\circ} \mathrm{C}$. The hydrodynamic diameter of THPP-HPC-55 changed from 180 to $200 \mathrm{~nm}$ in a reversible manner (Figure $2 c$ ), and similar changes in size were found in other HPC systems. ${ }^{24}$ In contrast, the hydrodynamic diameters of THPP-HPC-4 decreased during the initial heating process. Afterward, their size reversibly changed as that of THPP-HPC55 did. We further examined the photochemical properties of encapsulated THPP by measuring their fluorescence spectra. In both the systems, the fluorescence intensity of THPP increased during the heating process and decreased during the cooling process. In addition, these changes were reversible (Figure 2d). These changes in the fluorescence intensity of encapsulated THPP may have been due to the changes in the hydrophobicity of HPC. In contrast, THPP-Pul-55 formed large aggregates (over $1 \mu \mathrm{m}$ ) during the initial heating process (Figure S6), and the cooling process did not bring their hydrodynamic diameter back to the initial size. Moreover, the absorbance of THPP gradually decreased during the thermal cycling processes, and 50\% of THPP ultimately precipitated (Figure S7). These results suggested that HPC systems significantly improved the thermal stability of this hydrophobic drug in aqueous media compared to the non-thermoresponsive pullulan systems.

We attribute these thermoresponsive changes in the photochemical properties of THPP-HPC complexes to the changes in the hydrophobicity of the polymer matrix in HPC through pyrene. The ratio of the fluorescence intensities of pyrene at the emission wavelengths of $375\left(I_{375}\right)$ and $385 \mathrm{~nm}$ $\left(I_{385}\right)\left(I_{375} / I_{385}\right)$ is known as an indicator of the polarity of a microenvironment. ${ }^{30}$ Dissociated pyrene in a polar medium such as water results in an $I_{375} / I_{385}$ value of 1.75 . In contrast, the $I_{375} / I_{385}$ ratio of HPC at 25 and $55^{\circ} \mathrm{C}$ were 1.2 and 1.15 , respectively (Figure S8). These values indicate that HPC possesses a hydrophobic nanodomain within the polymer matrix, and its hydrophobicity increased during the heating process. In addition, the changes were reversible upon heating/ cooling cycling and fluorescence studies of THPP. Interestingly, excimer emission from pyrene gradually decreased upon thermal cycling (Figure S9), indicating that the heating process helps formulate the monomers of pyrene. ${ }^{31}$ These results supported our hypothesis of the manipulation of the photochemical properties of THPP within HPC. In addition, we investigated the thermal stimuli-triggered release profile of THPP in the presence of $10 \%$ fetal bovine serum (FBS) ${ }^{21}$ The dispersion of THPP-HPC-4 and THPP-HPC-55 in a medium containing $10 \%$ FBS was maintained at 4,37 , and $50{ }^{\circ} \mathrm{C}$, and fluorescence from released THPP was measured at each time point (Figure 2e). With increasing incubation temperatures, complexed THPP was more efficiently released from HPC in both the systems. More rapid payload release was found with THPP-HPC-4 than with THPP-HPC-55 at all the temperatures. At $24 \mathrm{~h}$ incubation at $50{ }^{\circ} \mathrm{C}$ mimicking the thermal condition surrounding the mitochondria, ${ }^{25}$ THPP-HPC-4 and THPP-HPC-55 released $26 \%$ and $22 \%$ of THPP, respectively. The cargo release may be caused by structural transition of the complex via thermal stimuli. Released THPP can bind serum proteins $^{32}$ or lipid vesicles, ${ }^{33}$ which are the major components of FBS. These differences in payload release may be due to highly packed THPP within the HPC polymer networks and their size-changing behavior upon thermal stimuli. The microenvironment around mitochondria is maintained at 50 ${ }^{\circ} \mathrm{C}$ because of the energy production process. ${ }^{19}$ The temper- 
ature-sensitive release of cargo molecules is a powerful means to attain mitochondria-targeted delivery by responding to the physiologically relevant levels of heat surrounding the mitochondria. In addition, encapsulated THPP was not released by acidic $\mathrm{pH}$, suggesting that our system can stably retain the cargo in the acidic tumor microenvironment and in endosomes (Figure S10).

The performance of the THPP-HPC complex as a photosensitizer was evaluated under visible light irradiation $\left(>620 \mathrm{~nm}\right.$ ) as measured by the generation of singlet oxygen. ${ }^{34}$ A time course of singlet oxygen generation was monitored by the quenching of the anthracene derivative ABDA into endoperoxide (Scheme S1). As singlet oxygen oxidized ABDA, the absorbance peaks at 360, 380, and $400 \mathrm{~nm}$ decreased, as shown in Figure S11. THPP-HPC-4 efficiently generated singlet oxygen compared to THPP-HPC-55 (Figure 2f). This may be because THPP-HPC-55 trapped a larger amount of THPP in each agglomerate, suggesting that encapsulated THPP was highly packed into a polymer, resulting in inactivation. ${ }^{35}$

Mitochondria-Targeted Delivery. We demonstrated the mitochondria-targeted delivery of THPP in murine colon carcinoma cells (Colon26) using THPP-Pul-55, THPP-HPC4, and THPP-HPC-55. To visualize the mitochondria, we used MitoGreen, which is a commercially available mitochondriastaining reagent. The subcellular distribution of mitochondria and complexes was observed by CLSM. In the case of THPPPul-55 and THPP-HPC-4, scant yellow pixels (the overlap of THPP with the mitochondria) were detected in the cytosol, indicating that delivered THPP (red pixels) were poorly overlapped with the mitochondria (green pixels) (Figure 3a,b). In contrast, the fluorescence signals from THPP-HPC-55 were highly overlapped with the mitochondria (Figure 3c). After 24 $\mathrm{h}$ incubation, the colocalization ratios of delivered THPP-Pul55, THPP-HPC-4, and THPP-HPC-55 with the mitochondria were 28,27 , and $67 \%$, respectively (Figure S12). These results indicate that THPP-HPC-55 was more suitable as a mitochondria-selective delivery platform for response to the mitochondrial heating process, as expected. In addition, the efficient accumulation of THPP in the mitochondria may enhance photoinduced cytotoxicity in cancer cells. To clarify these differences in the subcellular distribution of the delivered THPP between THPP-HPC-4 and THPP-HPC-55, we further examined the colocalization of delivered THPP with lysosomes, which plays a role in the digestion of exogenous materials in cells. With THPP-HPC-4, yellow pixels (overlap of delivered THPP with lysosomes) were detected in the cytosol (Figure S13a) at $4 \mathrm{~h}$, indicating that a large portion of THPP was trapped in lysosomes. Furthermore, a large part of the delivered THPP was still trapped by the lysosomes after $24 \mathrm{~h}$ (Figure S13b). This suggests that a large portion of the delivered THPP was inactivated via undesirable degradation in lysosomes. In contrast, THPP-HPC-55 was able to escape from lysosomes and spread through the cytosol (Figure $S 13 c, d)$. The differences in the endosomal escape may be due to the smaller size of THPP-HPC- $55^{36}$ and the large polydispersity of THPP-HPC-4 in size distribution. In case of both the systems, over $80 \%$ of the delivered THPP was trapped by the lysosomes during $4 \mathrm{~h}$ incubation (Figure S14a). After $24 \mathrm{~h}$ incubation, the colocalization ratios of the fluorescence signals from the delivered THPP in lysosomes using THPP-HPC-4 and THPP-HPC-55 were 66 and 18\%, respectively (Figure $\mathrm{S} 14 \mathrm{~b}$ ). These results suggest that excellent
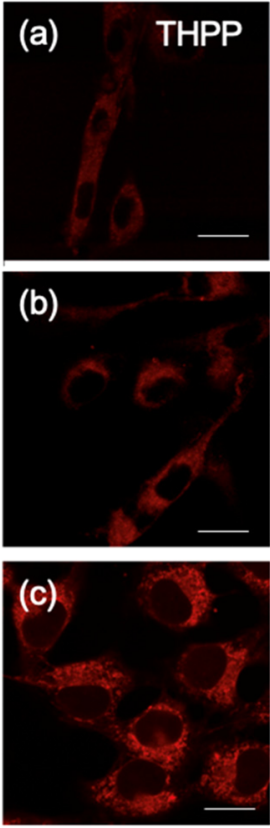

(d)
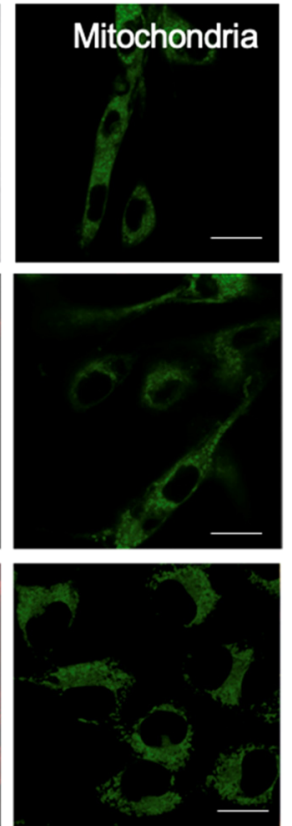

(e)
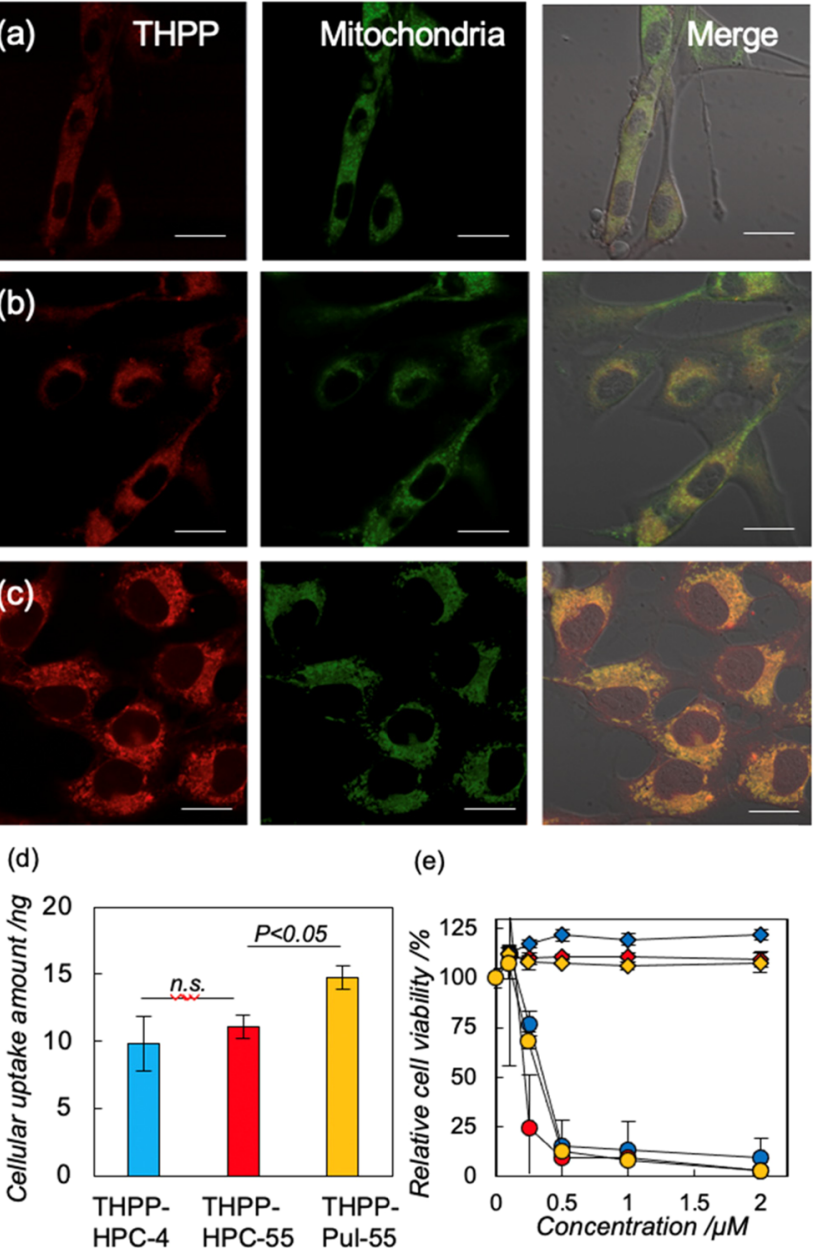

Figure 3. Photodynamic activities against a cancer cell line. $(a-c)$ Colocalization of delivered THPP-Pul-55 (a), THPP-HPC-4 (b), or THPP-HPC-55 (c) with the mitochondria. After $24 \mathrm{~h}$ incubation with a THPP-polysaccharide complex (red), the mitochondria were stained with MitoGreen (green). The samples were observed with confocal laser scanning microscopy (CLSM). The scale bars represent $20 \mu \mathrm{m}$. (d) Cellular uptake of THPP. HeLa cells were exposed to a THPP-Pul-55, THPP-HPC-4, or THPP-HPC-55 for $24 \mathrm{~h}$. Then, the cell lysates were prepared with RIPA buffer. The delivered THPP was extracted with the smallest amount of chloroform; its fluorescence was measured using a fluorometer $(N=3)$. The data were presented as mean $\pm S D$. The data were analyzed with the Student's $t$-test (twosided test). (e) Photoirradiation-induced anticancer effects on Colon 26 cells. Colon26 cells were coincubated with THPP-Pul-55 (yellow), THPP-HPC-4 (blue), or THPP-HPC-55 (red) for $24 \mathrm{~h}$. Subsequently, the cells were irradiated with red light for $30 \mathrm{~min}$ (circle). After an additional $24 \mathrm{~h}$, the cell viability was measured with the WST-8 assay $(N=3)$. The data were presented as mean \pm SD.

deliverability to the mitochondria was attained with THPPHPC-55 via efficient endosomal escape. Moreover, the photoinduced cytotoxicity of THPP from THPP-HPC-55 may be enhanced by inducing oxidative damage to the mitochondria in cancer cells, as shown in Scheme S2. ${ }^{37}$ In case of THPP-Pul-55, the colocalization ratio between the delivered THPP to lysosome decreased with the incubation time (Figure $\mathrm{S} 13 \mathrm{e}, \mathrm{S} 13 \mathrm{f})$. In contrast, the ratio between the delivered THPP to the mitochondria was still low $(28 \%)$. These results suggest that HPC worked as the mitochondria-targeting platform because of their thermoresponsiveness. To evaluate the deliverability of THPP, we quantified the cellular uptake of 
THPP using THPP-Pul-55, THPP-HPC-4, and THPP-HPC55 by measuring the fluorescence intensity of THPP extracted from the cells (Figure 3d). After $24 \mathrm{~h}$ incubation, THPP-Pul55 delivered a larger amount of THPP $\left(14 \mathrm{ng} / 1.0 \times 10^{5}\right.$ cells $)$ toward the Colon 26 cells than THPP-HPC-55 (12 ng/1.0 $\times$ $10^{5}$ cells). In addition, no obvious differences in the cellular uptake were found between THPP-HPC-4 and THPP-HPC$55\left(12 \mathrm{ng} / 1.0 \times 10^{5}\right.$ cells $)$.

We demonstrated the mitochondria-targeted delivery of THPP in murine colon carcinoma cells (Colon26) using THPP-Pul-55, THPP-HPC-4, and THPP-HPC-55. To visualize the mitochondria, we used MitoGreen, which is a commercially available mitochondria-staining reagent. The subcellular distribution of mitochondria and complexes was observed by CLSM. In the case of THPP-Pul-55 and THPPHPC-4, scant yellow pixels (the overlap of THPP with the mitochondria) were detected in the cytosol, indicating that delivered THPP (red pixels) were poorly overlapped with the mitochondria (green pixels) (Figure $3 \mathrm{a}, \mathrm{b}$ ). In contrast, the fluorescence signals from THPP-HPC-55 were highly overlapped with the mitochondria (Figure 3c). After $24 \mathrm{~h}$ incubation, the colocalization ratios of delivered THPP-Pul55, THPP-HPC-4, and THPP-HPC-55 with the mitochondria were 28,27 , and $67 \%$, respectively (Figure S12). These results indicate that THPP-HPC-55 was more suitable as a mitochondria-selective delivery platform for response to the mitochondrial heating process, as expected. In addition, the efficient accumulation of THPP in the mitochondria may enhance photoinduced cytotoxicity in cancer cells. To clarify these differences in the subcellular distribution of the delivered THPP between THPP-HPC-4 and THPP-HPC-55, we further examined the colocalization of delivered THPP with lysosomes, which plays a role in the digestion of exogenous materials in cells. With THPP-HPC-4, yellow pixels (overlap of delivered THPP with lysosomes) were detected in the cytosol (Figure S13a) at $4 \mathrm{~h}$, indicating that a large portion of THPP was trapped in lysosomes. Furthermore, a large part of the delivered THPP was still trapped by the lysosomes after $24 \mathrm{~h}$ (Figure S13b). This suggests that a large portion of the delivered THPP was inactivated via undesirable degradation in lysosomes. In contrast, THPP-HPC-55 was able to escape from lysosomes and spread through the cytosol (Figure $\mathrm{S} 13 \mathrm{c}, \mathrm{d})$. The differences in the endosomal escape may be due to the smaller size of THPP-HPC- $55^{36}$ and the large polydispersity of THPP-HPC-4 in size distribution. In case of both the systems, over $80 \%$ of the delivered THPP was trapped by the lysosomes during $4 \mathrm{~h}$ incubation (Figure S14a). After $24 \mathrm{~h}$ incubation, the colocalization ratios of the fluorescence signals from the delivered THPP in lysosomes using THPP-HPC-4 and THPP-HPC-55 were 66 and 18\%, respectively (Figure $\mathrm{S} 14 \mathrm{~b}$ ). These results suggest that excellent deliverability to the mitochondria was attained with THPPHPC-55 via efficient endosomal escape. Moreover, the photoinduced cytotoxicity of THPP from THPP-HPC-55 may be enhanced by inducing oxidative damage to the mitochondria in cancer cells, as shown in Scheme S2. ${ }^{37}$ In case of THPP-Pul-55, the colocalization ratio between the delivered THPP to lysosome decreased with the incubation time (Figure $\mathrm{S} 13 \mathrm{e}, \mathrm{S13f}$ ). In contrast, the ratio between the delivered THPP to the mitochondria was still low (28\%). These results suggest that HPC worked as the mitochondria-targeting platform because of their thermoresponsiveness. To evaluate the deliverability of THPP, we quantified the cellular uptake of
THPP using THPP-Pul-55, THPP-HPC-4, and THPP-HPC55 by measuring the fluorescence intensity of THPP extracted from the cells (Figure 3d). After $24 \mathrm{~h}$ incubation, THPP-Pul55 delivered a larger amount of THPP $\left(14 \mathrm{ng} / 1.0 \times 10^{5}\right.$ cells $)$ toward the Colon 26 cells than THPP-HPC-55 (12 ng/1.0 $\times$ $10^{5}$ cells). In addition, no obvious differences in the cellular uptake were found between THPP-HPC-4 and THPP-HPC$55\left(12 \mathrm{ng} / 1.0 \times 10^{5}\right.$ cells $)$.

Photodynamic Activity Against Cancer Cell Lines. We finally demonstrated the performance of the THPP-HPC complex as a photosensitizer for PDT in the Colon26 cells under visible light irradiation $(>620 \mathrm{~nm})$. The Colon 26 cells were incubated with the THPP-HPC systems for $24 \mathrm{~h}$. The cells were irradiated for $30 \mathrm{~min}(9 \mathrm{~mW})$, and cell viability was determined after $24 \mathrm{~h}$ incubation using a WST-8 assay. Under dark conditions, no apparent cytotoxicity was detected in these cell lines (Figure 3e, diamond symbol), and we confirmed that cell destruction was not induced by photoirradiation without exposing the THPP complex (Figure S15). In contrast, cell viability was significantly decreased with the increasing concentration of THPP under irradiation with visible light (Figure $3 \mathrm{e}$, circle symbol). In addition, the $\mathrm{IC}_{50}$ values using THPP-HPC-55 and THPP-HPC-4 were 0.21 and $0.36 \mu \mathrm{M}$, respectively. This indicates that THPP-HPC-55 showed enhanced photoinduced cytotoxicity in the Colon 26 cells with efficient mitochondrial-targeted delivery of THPP compared to THPP-HPC-4. Furthermore, the efficiencies in killing cancer cells using HPC systems were higher than those in killing cancer cells by the non-thermoresponsive group of THPP-Pul-55 $\left(\mathrm{IC}_{50}, 0.33 \mu \mathrm{M}\right.$; Figure 3e), although the deliverability of pullulan was higher than that of HPC. This suggests that mitochondrial targeting based on thermoresponsiveness is a powerful technique in PDT. We further measured the availability of THPP-HPC-55 as a photosensitizer in a human cervical cancer cell line (HeLa cell). Photoinduced cytotoxicity was also detected in the HeLa cells at concentrations similar to that which was effective in the Colon 26 cells (Figure S16). The $\mathrm{IC}_{50}$ value for THPP-HPC-55 in the HeLa cells was $0.11 \mu \mathrm{M}$, and photoinduced cytotoxicity was improved over free THPP $\left(\mathrm{IC}_{50}, 0.37 \mu \mathrm{M}\right)^{33}$ and the THPP/TMe- $\beta$-CD complex $\left(\mathrm{IC}_{50}, 0.15 \mu \mathrm{M}\right) .{ }^{13}$ Furthermore, the $\mathrm{IC}_{50}$ value of THPP-HPC-55 was 27 times higher than that of photofrin $(3.02 \mu \mathrm{M})$, which is a clinically available porphyrin oligomer. ${ }^{15}$ Moreover, current systems did not show severe cytotoxicity against red blood cells even at $1 \mu \mathrm{M}$ of THPP after the irradiation of light (Figure S17). This suggests that HPC could be used safely as nanoplatforms for PDT even under the irradiation of light.

To elucidate the mechanism of photoinduced cell death, we evaluated apoptosis detection assay using the Annexin-V/PI method. Flowcytometry (FACSCalibur, BECTON DICKINSON, New Jersey, USA) results revealed that all the systems induced late-apoptosis or necrosis via photoirradiation, and no obvious differences between these three systems were found during cell death (Figure S18). Then, we further examined the oxidative damages toward the mitochondrial membrane by detecting the changes in the mitochondrial membrane potential using JC-1 staining. In the normal mitochondria, the JC-1 staining emits red fluorescence via aggregate formation in response to the high membrane potential. The red fluorescence turns green when the mitochondria is damaged. ${ }^{38}$ While the photoinduced change in JC-1 fluorescence was slight in cells treated with THPP- 
HPC-4 or THPP-Pul-55 (Figure $4 \mathrm{a}-\mathrm{d}$ ), the photoinduced change in JC-1 fluorescence in the cells treated with THPP-
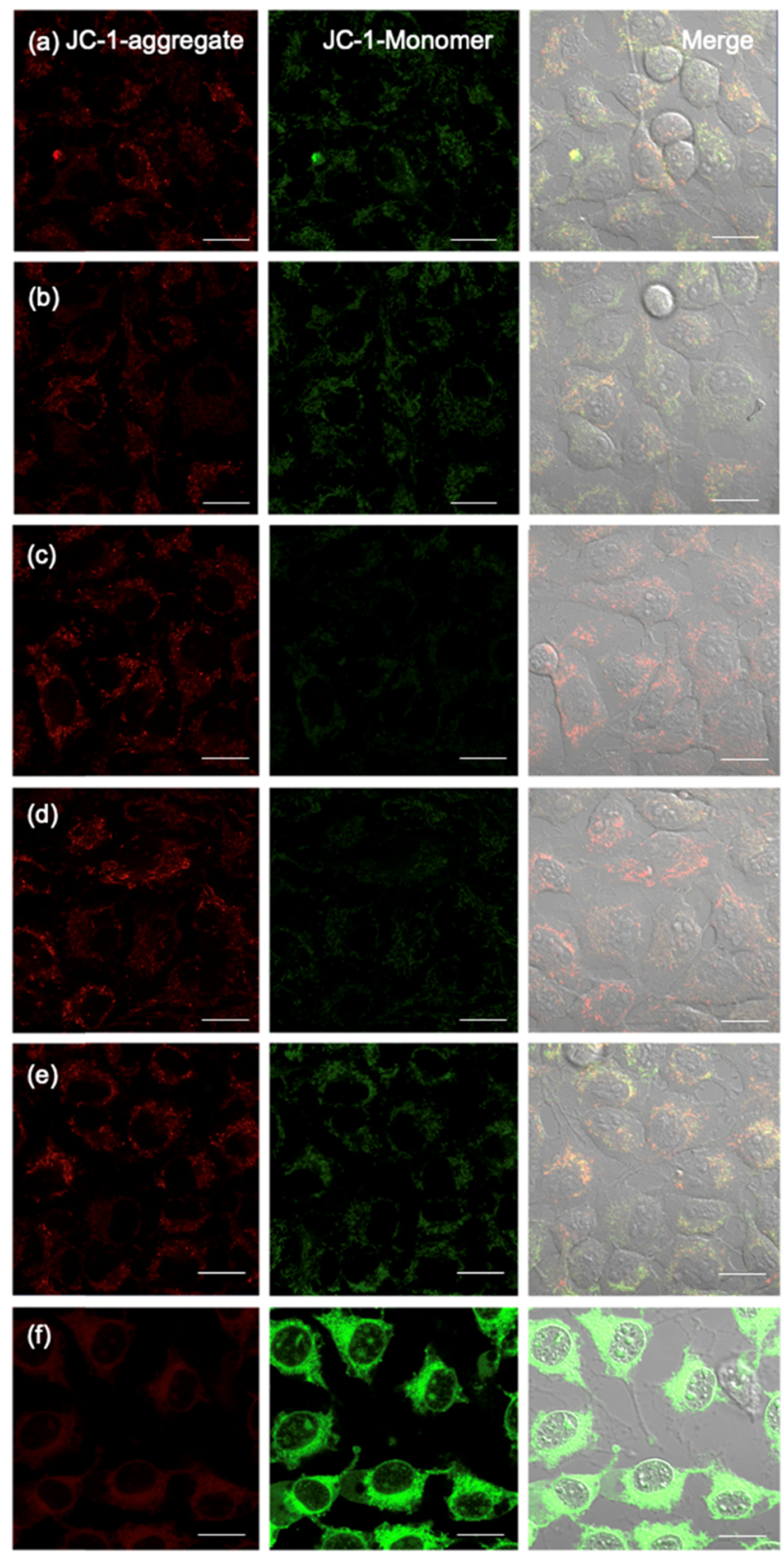

Figure 4. Mitochondrial dysfunction induced by photoirradiation. Colon26 cells treated with THPP-Pul-55 (before photoirradiation, (a); after photoirradiation, (b)), THPP-HPC-4 (before photoirradiation, (c); after photoirradiation, (d)), or THPP-HPC-55 (before photoirradiation, (e); after photoirradiation, (f)) for $24 \mathrm{~h}$ (THPP, $0.8 \mu \mathrm{M}$ ) were stained with the JC-1 probe $(1.5 \mu \mathrm{M})$. The samples were observed by CLSM. The error bar represents $20 \mu \mathrm{m}$.

HPC-55 was significant (Figure 4e,f). In addition, the cells treated with THPP-HPC-55 showed the most robust green fluorescence signals and the largest changes in fluorescence (Figure S19). These results indicate that, as we expected, THPP-HPC-55 is most efficient in causing cell death via oxidative stress against the mitochondrial membrane.

\section{CONCLUSIONS}

In conclusion, we demonstrate facile delivery nanoplatforms for hydrophobic THPP delivery using HPC. The thermoresponsiveness of HPC enabled the manipulation of encapsulation behaviors and release profiles, which is physiologically relevant to the surroundings of mitochondria $\left(50{ }^{\circ} \mathrm{C}\right)$. Moreover, the complex of THPP with HPC prepared at 55 ${ }^{\circ} \mathrm{C}$ enhanced photoinduced cytotoxicity in cancer cells by the efficient mitochondria-selective release of THPP. As noted in this study, our strategy has great potential for use as a photodynamic therapy for cancer.

\section{EXPERIMENTAL METHODS}

Preparation of THPP/HPC Complexes. HPC (10 mg) and THPP $(1.4 \mathrm{mg}, 2 \mu \mathrm{mol})$ were placed in a vial for processing in a high-speed vibrating mill. The mixture of HPC and THPP was processed in the high-speed vibrating mill at 30 $\mathrm{Hz}$ for $20 \mathrm{~min}$. Afterward, the complex was extracted with milli-Q at various temperatures $\left(4,10,25\right.$, and $\left.55^{\circ} \mathrm{C}\right)$. The emulsions were sonicated using an ultrasonic bath (Branson Ultrasonics, Missouri, USA) at $180 \mathrm{~W}$ and $42 \mathrm{kHz}$ for $1 \mathrm{~h}$. The precipitates were removed by centrifugation $(4500 \mathrm{rpm}, 20$ min, $25^{\circ} \mathrm{C}$ ). The concentration of the complexed THPP was determined by measuring absorbance using a UV-vis spectrometer (3600 UV-vis-NIR spectrometers, Shimadzu, Kyoto, Japan).

Characterization of the THPP-HPC Complex. The hydrodynamic diameter $\left(D_{\text {hy }}\right)$ of the complex of THPP with HPC was measured using a DLS instrument (Zeta-sizer Nano ZS; Malvern, Malvern, UK). The PDI value was calculated by cumulant fitting. The zeta potential of the complex was measured by Zeta-sizer Nano ZS using capillary cells. Morphological observations were carried out using a transmission electron microscope (JEM-1400, JEOL Ltd. Co., Tokyo, Japan). The samples were cast on a hydrophilized, ultrathin, carbon-deposited $\mathrm{Cu}$ grid and incubated for 30 mins. Afterward, the samples were stained with ammoniummolybdate (3 wt \%). The stained samples were observed using a transmission electron microscope (acceleration voltage, 100 $\mathrm{keV})$.

Thermoresponsiveness of the THPP-HPC Complex. The prepared THPP-HPC complex was incubated with a heat controller. At each temperature point, UV-vis spectra, fluorescence spectra, and the hydrodynamic diameter of the complex were measured using a UV-vis spectrometer, fluorometer, and Zeta-sizer, respectively. In addition, repetition effects on these physicochemical properties were evaluated using the same methods.

Thermo-Stimuli-Triggered Payload Release. THPPHPC-4 or THPP-HPC-55 (THPP, $5 \mu \mathrm{M}$ ) was maintained in a medium containing $10 \%$ FBS. The solutions were incubated at 4, 37, and $55^{\circ} \mathrm{C}$, and the released THPP was isolated as a precipitate via centrifugation $\left(4{ }^{\circ} \mathrm{C}, 12000 \mathrm{~g}, 20 \mathrm{~min}\right)$. The collected THPP complexes were redispersed in ethyl acetate, and their fluorescence intensities were measured using a fluorometer.

pH-Triggered Payload Release. THPP-HPC-4 or THPP-HPC-55 (THPP, $5 \mu \mathrm{M}$ ) was maintained in a medium containing $10 \%$ FBS. The solutions were incubated at $4{ }^{\circ} \mathrm{C}$ at different $\mathrm{pH}$ values of 5.4, 6.4, or 7.4. The released THPP was isolated as a precipitate via centrifugation $\left(4{ }^{\circ} \mathrm{C}, 12000 \mathrm{~g}, 20\right.$ $\mathrm{min})$. The collected THPP complexes were redispersed in 
ethyl acetate, and their fluorescence intensities were measured using a fluorometer.

Singlet Oxygen Species Detection Assay Using ABDA. Singlet oxygen species detection was carried out using ABDA. Oxygen gas was passed through the dispersion of the THPP-HPC complex (THPP, $5 \mu \mathrm{M}$ ) for $30 \mathrm{~min}$. ABDA in dimethyl sulfoxide solution was added to the dispersion, and photoirradiation was carried out $\left(15 \mathrm{~W} \bullet \mathrm{cm}^{-2},>620 \mathrm{~nm}, 60\right.$ min). At each time point, the absorbance of ABDA was measured using a UV-vis spectrometer, and the breaching efficiency was determined by Abs/Abs ${ }_{0}$ at $400 \mathrm{~nm}$.

Photodynamic Activity Against Cancer Cell Lines. The Colon26 cells or HeLa cells were seeded on 48-well plates (Thermo Fischer Science) at $1.71 \times 10^{4}$ cells $(N=3)$ and incubated overnight (approximately $18 \mathrm{~h}$ ). The cells were exposed to the THPP-HPC complex or the THPP-Pul complex at varying concentrations. After $24 \mathrm{~h}$ incubation, the cells were washed with PBS thrice, and photoirradiation (610$740 \mathrm{~nm}, 9 \mathrm{~mW} \bullet \mathrm{cm}^{-2}$ ) was carried out for 30 mins. Afterward, the cells were incubated for $24 \mathrm{~h}$, and the Cell Counting Kit- 8 solution was added to the cells. After $30 \mathrm{~min}$ of incubation, absorbance at $450 \mathrm{~nm}$ was measured using a microplate reader.

Quantification of the Cellular Uptake of THPP. The Colon26 cells were seeded on 12-well plates (Thermo Fischer Science) at $1.0 \times 10^{5}$ cells per well $(N=3)$ and incubated overnight (approximately $18 \mathrm{~h}$ ). The cells were exposed to the THPP-HPC complex (THPP, $0.8 \mu \mathrm{M}$ ). After $24 \mathrm{~h}$ incubation, the cells were washed with PBS thrice. The cells were lysed with RIPA buffer (Fujifilm, Tokyo, Japan) and extracted with ethylacetate. The fluorescence intensities of THPP were measured using a fluorometer.

Subcellular Distribution of the Delivered THPP. The Colon26 cells were seeded on glass-bottom dishes (Iwaki, Tokyo, Japan) at $1.0 \times 10^{5}$ cells per dish and incubated overnight (approximately $18 \mathrm{~h}$ ). The cells were exposed to the THPP-HPC complex (THPP, $0.8 \mu \mathrm{M}$ ). After $24 \mathrm{~h}$ incubation, the cells were washed with PBS thrice. Lysosomes and mitochondria were stained using commercially available fluorescent reagents, Lysotracker green or MitoGreen. The samples were observed by CLSM (LSM700, Carl Zeiss, Germany).

Mechanisms of Photoinduced Cell Death. The changes in the mitochondrial membrane potential after photoirradiation were detected by JC-1 staining. The Colon 26 cells were seeded on the bottom of a glass dish at $1 \times 10^{5}$ cells/dish and incubated overnight. The cells were incubated with THPP-Pul55, THPP-HPC-4, or THPP-HPC-55 (THPP, $0.8 \mu \mathrm{M}$ ). After $24 \mathrm{~h}$ incubation, the cells were irradiated at $610-740 \mathrm{~nm}, 9$ $\mathrm{mW} \bullet \mathrm{cm}^{-2}$ for $30 \mathrm{~min}$. Afterward, the cells were stained with JC-1 $(1.5 \mu \mathrm{M})$ and observed using CLSM.

Statistical Analysis. All results are presented as mean \pm SD. The variance analysis was performed with Student's $t$-test (two-sided test). A significant p-value indicates a significant difference, where the probability is less than $0.05(*), 0.01$ $(* *)$, and $0.001(* * *)$. The analysis was carried out based on excel statistics.

\section{ASSOCIATED CONTENT}

\section{SI Supporting Information}

The Supporting Information is available free of charge at https://pubs.acs.org/doi/10.1021/acsomega.0c05647.
${ }^{1} \mathrm{H}-\mathrm{NMR}$ spectrum of $\mathrm{HPC}$ in d6-DMSO; transmittance at $500 \mathrm{~nm}$ of $1 \mathrm{mg} / \mathrm{mL} \mathrm{HPC}$ in water; UV-vis spectra of THPP-HPC-4 (blue), THPP-HPC-10 (green), THPP-HPC-25 (yellow), THPP-HPC-40 (purple), and THPP-HPC-55 (red) redissolved in DMSO; size distribution of THPP-HPC-4 and THPP-HPC-55; size distribution of the complexes was analyzed by ImageJ using 35 samples; UV-vis spectra of THPP-Pul-4 (blue), THPP-Pul-25 (yellow), and THPP-Pul-55 (red) in DMSO; properties of the THPP-Pul complexes in solutions prepared at various temperatures; changes in hydrodynamic diameters in THPP-Pul-55 during the thermal process (blue, $25{ }^{\circ} \mathrm{C}$; red, $55{ }^{\circ} \mathrm{C}$ ); thermal stability of THPP-Pul-55; changes in the UV-vis spectrum of THPP-Pul-55 during the thermal process; stability of the complex of THPP with pullulan during the thermal cycle process $\left(25{ }^{\circ} \mathrm{C}\right.$, blue; $55{ }^{\circ} \mathrm{C}$, red); stability was calculated as the relative absorbance at each point to the absorbance at the initial data point at 420 $\mathrm{nm}$; I375/I385 ratio of pyrene-HPC-25 during thermal cycling from $25{ }^{\circ} \mathrm{C}$ (blue) to $55{ }^{\circ} \mathrm{C}$ (red); changes in the fluorescence spectrum of pyrene-HPC-25 during thermal cycling from $25{ }^{\circ} \mathrm{C}$ (blue) to $55{ }^{\circ} \mathrm{C}$ (red); $\mathrm{pH}$ triggered payload release of THPP-HPC-4 (circle) and THPPHPC-55 (diamond); conversion of ABDA to endoperoxide via oxidation by ${ }^{1} \mathrm{O}_{2}$; representative UV absorption spectrum before and after the bleaching of ABDA; colocalization ratio of mitochondria to the delivered THPP; subcellular distribution of the delivered THPP-HPC-4; schematic illustration of the mechanism underlying photoinduced cytotoxicity; anticancer effects via photoirradiation toward Colon26 cells and HeLa cells; anticancer effects via photoirradiation in HeLa cells; hemolysis assay with irradiation of light; apoptosis detection assay using the annexinV/PI method; and measuring photoirradiation-induced mitochondrial membrane potential changes using the JC-1 probe (PDF)

\section{AUTHOR INFORMATION}

\section{Corresponding Authors}

Riku Kawasaki - Program of Applied Chemistry, Graduate School of Advanced Science and Engineering, Hiroshima University, Higashi Hiroshima 739-8527, Japan; ○ orcid.org/0000-0003-1621-8189; Email: riku0528@ hiroshima-u.ac.jp

Atsushi Ikeda - Program of Applied Chemistry, Graduate School of Advanced Science and Engineering, Hiroshima University, Higashi Hiroshima 739-8527, Japan; 다이.org/0000-0003-3492-3455; Email: aikeda@ hiroshima-u.ac.jp

\section{Authors}

Keita Yamana - Program of Applied Chemistry, Graduate School of Advanced Science and Engineering, Hiroshima University, Higashi Hiroshima 739-8527, Japan

Risako Shimada - Program of Applied Chemistry, Graduate School of Advanced Science and Engineering, Hiroshima University, Higashi Hiroshima 739-8527, Japan

Kouta Sugikawa - Program of Applied Chemistry, Graduate School of Advanced Science and Engineering, Hiroshima University, Higashi Hiroshima 739-8527, Japan 
Complete contact information is available at:

https://pubs.acs.org/10.1021/acsomega.0c05647

\section{Author Contributions}

${ }^{\ddagger}$ R.K. and K.Y. contributed equally. The manuscript was written through contributions of all authors. All authors have given approval to the final version of the manuscript.

\section{Funding}

This work was supported by JSPS KAKENHI Grant-in-Aid for Scientific research (B) (A.I., Grant No. JP16H04133), EarlyCareer Scientists (K.S., Grant No. 19 K15523; R.K., Grant No. 19 K15401), and foundation from Kyoto Technology and Science Center (R.K.).

\section{Notes}

The authors declare no competing financial interest.

\section{ACKNOWLEDGMENTS}

The experiments using CLSM and TEM were carried out at the Natural Science Center for Basic Research and Development, Hiroshima University. The author would like to thank Enago (www.enago.jp) for the English language review.

\section{REFERENCES}

(1) Dolmans, D. E. J. G. J.; Fukumura, D.; Jain, R. K. Photodynamic Therapy for Cancer. Nat. Rev. Cancer 2003, 3, 380-387.

(2) Lu, Y.; Li, L.; Lin, Z.; Wang, L.; Lin, L.; Li, M.; Zhang, Y.; Yin, Q.; Li, Q. Photodynamic Therapy: A New Treatment Modality for Rheumatoid Arthritis: Combined Photothermal and Photodynamic Therapy Using Cu7.2S4 Nanoparticles. Adv. Healthcare Mater. 2018, 7,1800013

(3) Li, X.; Kolemen, S.; Yoon, J.; Akkaya, E. U. Activatable Photosensitizers: Agents for Selective Photodynamic Therapy. Adv. Funct. Mater. 2017, 27, 1604053.

(4) Hamblin, M. R. Fullerene as Photosensitizers in Photodynamic Therapy: Pros and Cons. Photochem. Photobiol. Sci. 2018, 17, 15151533.

(5) Ethirajan, M.; Chen, Y.; Joshi, P.; Pandey, R. K. The Role of Porphyrin chemistry in tumor imaging and Photodynamic Therapy. Chem. Soc. Rev. 2011, 40, 340-362.

(6) Lo, P. C.; Morgade, M. S. R.; Pandey, R. K.; Ng, D. K. P.; Torres, T.; Dumoulin, F. The Unique Feature and Promises of Phthalocyanines as Advanced Photosensitizers for Photodynamic Therapy of Cancer. Chem. Soc. Rev. 2020, 49, 1041-1056.

(7) Rapp, T. L.; DeForest, C. A. Visible Light-Responsive Dynamic Biomaterials: Going Deeper and Triggering More. Adv. Healthcare Mater. 2020, 9, 1901553.

(8) Rabiee, N.; Yaraki, M. T.; Garakani, S. M.; Garakani, S. M.; Ahmadi, S.; Lajevardi, A.; Baghezadeh, M.; Rabiee, M.; Tayebi, L.; Tahriri, M.; Hamblin, M. R. Recent Advances in Porphyrin-based Composites for Effective Targeted Imaging and Therapy. Biomaterials 2020, 232, 119707.

(9) Tian, B.; Hua, S.; Liu, J. Cyclodextrin-based Delivery Systems for Chemotherapeutic Anticancer Drugs: A Review. Carbohydrate Polym. 2020, 232, 115805.

(10) Kadhim, A.; McKenzie, L. K.; Bryant, H. E.; Twyman, L. J. Synthesis and Aggregation of A Porphyrin-cored Hyperbranched Polyglycidol and Its Application as A Macromolecular Photosensitizer for Photodynamic Therapy. Mol. Pharmaceutics 2019, 16, 1132-1139.

(11) Webber, M. J.; Langer, R. Drug Delivery by Supramolecular Design. Chem. Soc. Rev. 2017, 46, 6600-6620.

(12) Webber, M. J.; Appel, E. A.; Meijer, E. W.; Langer, R. Supramolecular Biomaterials. Nat. Mater. 2016, 15, 13-26.

(13) Ikeda, A.; Satake, S.; Mae, T.; Ueda, M.; Sugikawa, K.; Shigeto, H.; Funabashi, H.; Kuroda, A. Photodynamic Activities of Porphyrin Derivative-Cyclodextrin Complexes by Photoirradiation. ACS Med. Chem. Lett. 2017, 8, 555-559.
(14) Hino, S.; Funada, R.; Sugikawa, K.; Koumoto, K.; Suzuki, T.; Nagasaki, T.; Ikeda, A. Turn-on Fluorescence and Photodynamic Activity of $\beta$-(1,3-1,6)-D-Glucan-complexed Porphyrin Derivatives inside HeLa Cells. Photochem. Photobiol. Sci. 2019, 18, 2854-2858.

(15) Yamana, K.; Kawasaki, R.; Sugikawa, K.; Ikeda, A. Solubilization of Tetrahydroxyphenylchlorin in Water and Improved Photodynamic Activity after Complexation with Cyclic Oligo- and Polysaccharides. ACS Appl. Bio Mater. 2020, 3, 3217-3225.

(16) Nakaya, T.; Horiguchi, B.; Hino, S.; Sugikawa, K.; Funabashi, H.; Kuroda, A.; Ikeda, A. Stabilization of Lipid Membraneincorporated Porphyrin Derivative Aqueous Solutions and Their Photodynamic Activities. Photochem. Photobiol. Sci. 2019, 18, 459466.

(17) Lv, W.; Zhang, Z.; Zhang, K. Y.; Yang, H.; Liu, S.; Xu, A.; Guo, S.; Zhao, Q.; Huang, W. A Mitochondria-Targeted Photosenstizer Showing Improved Photodynamic Therapy Effects under Hypoxia. Angew. Chem., Int. Ed. 2016, 55, 9947-9951.

(18) Thomas, A. P.; Palanikumar, L.; Jeena, M. T.; Kim, K.; Ryu, J. H. Cancer-Mitochondria-Targeted Photodynamic Therapy with Supramolecular Assembly of HA and a Water Soluble NIR Cyanine Dye. Chem. Sci. 2017, 8, 8351-8356.

(19) Yang, X.; Wang, D.; Wang, J.; Xue, L.; Ou, C.; Wang, W.; Liu, M.; Song, X.; Dong, X. Functional Black Phosphorous Nanosheets for Mitochondria-Targeting Photothermal/Photodynamic Synergistic Cancer Therapy. Chem. Sci. 2019, 10, 3779-3785.

(20) Chuah, J. A.; Matsugami, A.; Hayashi, F.; Numata, K. SelfAssembled Peptide-Based System for Mitochondrial-Targeted Gene Delivery: Functional and Structural Insights. Biomacromolecules 2016, 17, 3547-3557.

(21) Wang, D.; Huang, H.; Zhou, M.; Lu, H.; Chen, J.; Chang, Y. T.; Gao, J.; Chai, G.; Hu, Y. A Thermoresponsive Nanocarrier for Mitochondria-Targeted Drug Delivery. Chem. Commun. 2019, 55, 4051-4054

(22) Wang, D.; Zhou, M.; Huang, H.; Ruan, L.; Lu, H.; Zhang, J.; Chen, J.; Gao, J.; Chai, Z.; Hu, Y. Gold Nanoparticle-Based Probe for Analyzing Mitochondrial Temperature in Living Cells. ACS Appl. Bio Mater. 2019, 2, 3178-3182.

(23) Bielska, D.; Karewicz, A.; Kaminski, K.; Kielkowicz, I.; Lachowicz, T.; Szczubialka, K.; Npwakowska, M. Self-Organized Thermo-Responsive Hydroxypropyl Cellulose Nanoparticles for Curcumin Delivery. Eur. Polym. J. 2013, 49, 2485-2494.

(24) Tahara, Y.; Sakiyama, M.; Takeda, S.; Nishimura, T.; Mukai, S.; Sawada, S.; Sasaki, Y.; Akiyoshi, K. Self-Assembled Nanogels of Cholesterol-Bearing Hydroxypropyl Cellulose: A Thermoresponsive Building Block for Nanogel Tectonic Materials. Langmuir 2016, 32, 12283-12289.

(25) Chretien, D.; Benit, P.; Ha, H. H.; Keipert, S.; Khoury, R. E.; Chang, Y. T.; Jastroch, M.; Jacobs, H. T.; Rustin, P.; Rak, M. Mitochondria Are Physiologically Maintained at Close to $50{ }^{\circ} \mathrm{C}$. PLoS Biol. 2018, 16, No. e2003992.

(26) Nguyen, T.; Latkany, R. Review of Hydroxypropyl Cellulose Ophthalmic Inserts for Treatment of Dry Eye. Clin. Ophthalmol. 2011, 5, 587-591.

(27) Horiguchi, B.; Nakaya, T.; Ueda, M.; Sugikawa, K.; Mizuta, T.; Haino, T.; Kawata, N.; Ikeda, A. Controllable Direction of Porphyrin Derivatives in Two Cyclodextrin Cavities. Eur. J. Org. Chem. 2018, 2018, 2138-2143.

(28) Ikeda, A.; Iizuka, T.; Maekubo, N.; Nobusawa, K.; Sugikawa, K.; Koumoto, K.; Suzuki, T.; Nagasaki, T.; Akiyama, M. Water Solubilization of Fullerene Derivatives by $\beta$-(1,3-1,6)-D-Glucan and Their Photodynamic Activities toward Macrophages. Chem. - Asian J. 2017, 12, 1069-1074.

(29) Hino, S.; Satake, S.; Shinmori, H.; Kawabata, S.; Koumoto, K.; Suzuki, T.; Nagasaki, T.; Sugikawa, K.; Kawasaki, R.; Ikeda, A. Improved Stability and Photodynamic Activity of Water-Soluble 5,15Diazaporphyrins Incorporated in $\beta$ - $(1,3-1,6)$-D-Glucan with On-Off Switch. Chem. - Asian J. 2019, 15, 365-370. 
(30) Kawasaki, R.; Sasaki, Y.; Akiyoshi, K. Self-Assembled Nanogels of Carborane-Bearing Polysaccharides for Boron Neutron Capture Therapy. Chem. Lett. 2017, 46, 513-515.

(31) Yadav, A.; Kurur, N. D.; Pandey, S. Intramolecular Excimer Formation Dynamics of 1,3-Bis(1-pyrenyl)propane within 1-butyl-3methylimidazolium hexafluorophosphate and Its Polyethylene Glycol Mixture. J. Phys. Chem. B 2015, 119, 13367-13378.

(32) Xiao, C. Q.; Jiang, F. L.; Zhou, B.; Li, R.; Liu, Y. Interaction between A Cationic Porphyrin and Bovine Serum Albumin Studied by Surface Plasmon Resonance Fluorescence Spectroscopy and Cyclic Voltammetry. Photochem. Photobiol. Sci. 2011, 10, 1110-1117.

(33) Yumoto, T.; Satake, S.; Hino, S.; Sugikawa, K.; Kawasaki, R.; Ikeda, A. Improved Water Solubility and Photodynamic Activity of Hydroxy-modified Porphyrins by Complexation with Cyclodextrin. Org. Biomol. Chem. 2020, 18, 6702-6709.

(34) Lindig, B. A.; Rodgers, M. A. J.; Schaap, A. P. Determination of The Lifetime of Singlet Oxygen in Water-d2 Using 9,10anthracenedipropionic acid, A Water-Soluble Probe. J. Am. Chem. Soc. 1980, 102, 5590-5593.

(35) Andrade, S. M.; Teixeira, R.; Costa, S. M. B.; Sobral, A. J. F. N. Self-Aggregation of Free Base Porphyrins in Aqueous Solution and in DMPC Vesicles. Biophys. Chem. 2008, 133, 1-10.

(36) Smith, S. A.; Selby, L. I.; Johnson, A. P. R.; Such, G. K. The Endosomal Escape of Nanoparticles: Toward More Efficient Cellular Delivery. Bioconjugate Chem. 2018, 30, 263-272.

(37) Morgan, J.; Oseroff, A. R. Mitochondria-based Photodynamic Anti-Cancer Therapy. Adv. Drug Delivery Rev. 2001, 49, 71-86.

(38) Reers, M.; Smith, T. W.; Chen, L. B. J-Aggregate Formation of A Carbocyanine as A Quantitative Fluorescent Indicator of Membrane Potential. Biochemistry 1991, 30, 4480-4486. 